\title{
Competitive interactions in Drosophila melanogaster: recurrent selection for aggression and response
}

\author{
Mortaza Hemmat and \\ Paul Eggleston
}

\author{
Department of Genetics, University of Liverpool, \\ P.O. Box 147, Liverpool L69 3BX, U.K.
}

\begin{abstract}
Recurrent selection programmes for both high response and low aggression have been employed in the Texas population of Drosophila melanogaster. Five main points have emerged from this investigation. First, the population exhibits extensive genetic variation for the aggression and response components of competitive interactions which take place in genetically heterogeneous cultures. Secondly, the two components of such interactions, namely aggression and response, can be adjusted by the selection of particular groups of genes. Thirdly, the rapid change in the selected components in the early generations suggests that a considerable amount of additive genetic variation is involved in the control of aggression and response. This is further supported by estimates of the realised heritability taken over the whole selection programme which amount to 0.79 and 0.74 for the low aggression and high response selections respectively. Fourthly, the rapidity with which changes in aggression and response approached plateaux suggests that such changes in the earlier generations are primarily due to the assortment of major chromosomes as units. Fifthly, it was concluded that aggression and response do not behave entirely independently or dependently. The results suggest that a single array of genes might be responsible for the determination of both characters. The observed results would then be consistent with certain of these genes having a much larger effect on aggression than they do on response.
\end{abstract}

\section{INTRODUCTION}

Recent advances in the quantification of competitive interactions in genetically heterogeneous mixtures have shown that both intra-genotypic and inter-genotypic effects may be estimated independently (Mather and Caligari, 1981). These competitive effects may be combined to describe how genotypes exert competitive pressure or aggression on the one hand, and respond to such aggression on the other. This distinction between aggression and response was drawn by Breese and Hill (1973) and the analytical procedure was refined by Mather and Caligari (1983). Subsequently, this model for the analysis of competitive interactions (although developed using Drosophila melanogaster as a model system) has been applied to a range of experimental and commercial material including Lolium perenne (Mather, Hill and Caligari, 1982) and Hordeum vulgare (Powell, Caligari and Thomas, 1985). In addition, the flexibility of this multiple linear regression approach has been demonstrated through a variety of experimental designs, including both substitution and addition techniques (de Miranda and Eggleston, 1987) and with a variety of cornpetitive characters (Eggleston, 1987; Powell et al., 1985).

All of these experiments have identified considerable amounts of genetical variation with respect to competitive interactions (Eggleston, 1985; de Miranda and Eggleston, 1987) and this supports earlier evidence for the heritability of competitive ability in genetical mixtures of Drosophila (Mather and Cooke, 1962; Gale, 1964), Hordeum (Sakai and Gotoh, 1955) and Tribolium (Lerner and Ho, 1961). In addition, the results of competition experiments between $D$. melanogaster and $D$. simulans (Moore, 1952) and those between wild type and dumpy wing mutants of $D$. melanogaster (Seaton and Antonovics, 1967) show clearly that competitive ability, in its broad sense, can be improved by selection.

The ability to quantify aggression and response parameters, however, has led to the suggestion that these components of the competitive interaction behave as independent metrical characters which are separately adjustable by selection (Mather and Caligari, 1983; Eggleston, 1985). Whilst such a situation may be defined in terms of independent genetical control, this is not a necessary 
prerequisite. It is possible that the genes which determine aggression, for example, are also involved in the control of response in such a way that the effects on the two characters are related, but not in any systematic fashion. Consequently, the actual relationship observed between aggression and response might take a variety of forms (Eggleston, 1985). The implications of selective independence for aggression and response, with respect to both experimental and commercial populations, are considerable. It may, for example, be possible to select crop species for the appropriate combinations of aggression and response in order to maximise yields or to minimise losses due to invading weed competitors.

The experiment reported in this paper represents the first attempt to independently adjust aggression and response levels in a genetically segregating population. The implications of these experiments and the inferred nature of the genetical control of aggression and response are discussed.

\section{EXPERIMENTAL DETAILS}

This experiment was carried out using progeny raised from eggs sampled from the Texas population of Drosophila melanogaster previously described by Barnes (1968). This cage population was routinely maintained at $18^{\circ} \mathrm{C}$ with a population size of approximately 2000 adults. The Texas population was chosen because it exhibits very high levels of aggression and very low levels of response. This is to be expected as a direct result of natural selection operating within the intensely competitive cage conditions (Eggleston, 1985). In addition a long inbred strain derived from the Texas population and denoted $\mathrm{y}^{2} \mathrm{~T} 25$ was used. This was created by the introduction of the body colour marker $\left(\mathrm{y}^{2}\right)$ into the wild type strain T25, as described by Mather and Caligari (1983). The introduction of this particular allele has a negligible effect on the competitive interactions (Eggleston, 1987) and makes it possible to distinguish the indicator and associate competitors in duoculture. Eggs were sampled from the Texas population by replacing a food vial, for a short time, with a vial containing a laying stick coated with blackened starch paste. These eggs were transferred to bottles and allowed to complete their development at $25^{\circ} \mathrm{C}$ in order to ensure sufficient adults for the quantity of eggs required. Upon emergence, these adults (representative of the range of genetical variation in the original Texas population) were mated inter se for 2 days and then allowed to lay eggs on blackened starch paste for 2 hours at $25^{\circ} \mathrm{C}$ in order to minimise developmental heterogeneity. Eggs were obtained in a similar way from the tester strain $y^{2} \mathrm{~T} 25$, and both groups of eggs were used to initiate the selection programme. It has previously been shown that the Texas population, despite its genetical heterogeneity, can be considered as a component in mixtures in exactly the same way as inbred genotypes (Eggleston, 1985). Throughout this paper, components derived from the Texas population are referred to as lines.

The design of the recurrent selection programme (fig. 1) was such that individuals from the Texas population were allowed to compete with the tester strain $\mathrm{y}^{2} \mathrm{~T} 25$ in order to establish the existing levels of aggression and response of the Texas population in combination with a specific tester genotype (cycle 0 ). Subsequently, a range of 18 lines were isolated from the population by single pair matings and the resulting progeny sets from these lines (which were expected to differ genetically) were allowed to lay eggs as described previously. These eggs were used to initiate competition experiments, such that individuals from each line competed independently with the tester

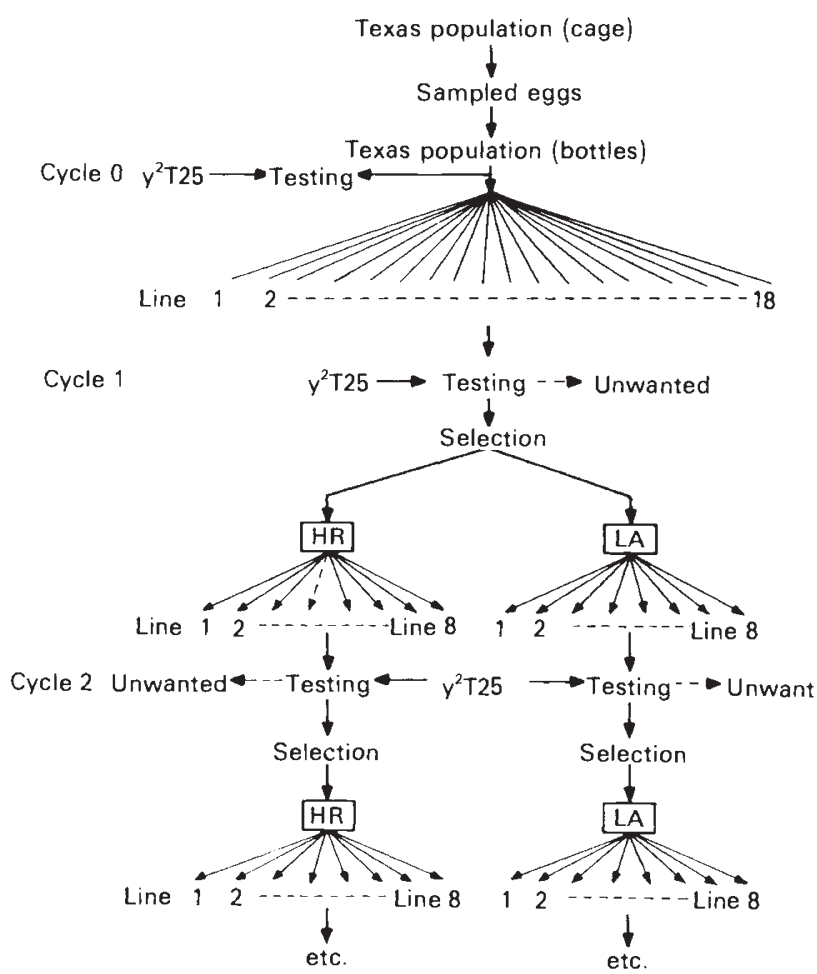

Figure 1 Diagrammatic scheme of selection and testing procedure. 
genotype $y^{2} T 25$. Because of the practical limitations of experimental size it was not possible to select for all combinations of aggression and response. Since the Texas population exhibited high aggression and low response it was decided that the most obvious changes would be seen by attempting to reverse this trend through selection for high response (HR) and low aggression (LA). On the basis of the cycle 0 competition experiments two lines were selected, namely those which had produced the largest increase in response and the largest decrease in aggression. These two lines then formed the basis of the high response (HR) and low aggression (LA) selection programmes. Within each selection programme subsequent rounds of selection were carried out as follows. As flies began to emerge from the duoculture competition tubes, wild type (Texas) males and females were separated upon emergence so as to ensure that the females remained virgin. These adults were then used to initiate eight single pair matings for each selection programme. The eggs from each of these single pair matings were then used independently in eight competition experiments against the tester strain $y^{2} \mathrm{~T} 25$. At no time did genes from the tester genotype $y^{2} \mathrm{~T} 25$ enter the Texas selection lines. As before, the results of these experiments were utilised to select the appropriate lines to continue each selection programme.

The investigation utilised a substitution or replacement design (de Wit, 1960) together with the regression analysis of Mather and Caligari (1981). Values of intra- and inter-genotypic competition were used to calculate aggression and response components as described by Mather and Caligari (1983). The indicator and associate genotypes were competed both individually as monocultures and together in duocultures. Each density series was set up using counted numbers of eggs. This was achieved by mating the flies of the different genotypes separately at $25^{\circ} \mathrm{C}$ for 2 days, before allowing them to lay for 2 hours at $25^{\circ} \mathrm{C}$ on plastic partitions coated with blackened starch paste in $3^{\prime \prime} \times 1^{\prime \prime}(2.5 \times 7.5 \mathrm{~cm})$ vials. This technique was used in order to minimise developmental heterogeneity. The required number of eggs were then picked off the starch stick with a sterilised needle and placed on the surface of previously prepared tubes $(2.5 \times 7.5 \mathrm{~cm})$, containing $5 \mathrm{ml}$ of 2 per cent Bacto-agar to which $15 \mathrm{ml} / \mathrm{l}$ Nipagin (10 per cent in ethanol) had been added as an anti-fungal agent. On to the surface of this medium $0.3 \mathrm{ml}$ of live yeast suspension was then dispensed. The original concentration of this suspension was $13.5 \mathrm{~g}$ dried bakers yeast (Sigma YSC-
2) in $90 \mathrm{ml}$ distilled water. Thus the amount of food available for the newly hatched competing larvae was $45 \mathrm{mg}$ of live yeast in each vial. The vials were then left 48 hours to dry at $25^{\circ} \mathrm{C}$. Each competition experiment consisted of the following density series where the figures represent the number of eggs seeded into the competition tubes;

\begin{tabular}{|c|c|c|c|c|c|}
\hline $\begin{array}{l}\text { Indicator } \\
\text { monocultures }\end{array}$ & 120 & 90 & 60 & 30 & \\
\hline $\begin{array}{l}\text { Associate } \\
\text { monocultures }\end{array}$ & & 30 & 60 & 90 & 120 \\
\hline $\begin{array}{l}\text { Indicator: Associate } \\
\text { duocultures }\end{array}$ & & $90: 30$ & $60: 60$ & $30: 90$ & \\
\hline
\end{tabular}

The monocultures with 120 eggs can be considered as the starting and finishing points of each duoculture series.

All competition experiments were replicated to provide an estimate of sampling variation. All cultures within each replicate were individually randomised and all replicates were raised at $25 \pm$ $0.5^{\circ} \mathrm{C}$. The percentage egg hatchability of each genotype was calculated prior to the competition experiments in order to determine any bias in the estimation of competitive effects between larvae as described by Caligari (1980). In all cases, the egg hatchabilities remained relatively consistent at around 90 per cent.

The character $P$, the proportion of eggs successfully developing into adults (Caligari, 1980) was chosen to measure competitive success. Each value of $P$ was transformed into an angle $\left(P_{a}\right)$ using the standard angular transformation (Fisher and Yates, 1963), in order to normalise sampling distributions.

The original analysis of Mather and Caligari (1981) was utilised throughout these experiments to estimate the intra- and inter-genotypic competitive effects in monoculture and duoculture respectively. As an example we can consider results obtained for the Texas population and $\mathrm{y}^{2} \mathrm{~T} 25$ in cycle 0 (reflecting the initial characteristics of the Texas population) which are depicted in fig. 2 . The slope with gradient $b_{m}$ obtained from the regression of $P_{a}$ on indicator genotype density in monoculture measures intra-genotypic competition. The magnitude of this slope is estimated as $\left(b_{0}-b_{m}\right)$ following the description of Mather et al., (1982), where $b_{0}$ is the line of zero relationship. A similar slope with gradient $b_{d}$ obtained from the duoculture density series, measures the effect of the associate competitors on the indicator genotype (inter-genotypic effect) in addition to the intragenotypic competition among indicator individuals. Consequently, the inter-genotypic effect of associate on indicator is estimated as the 

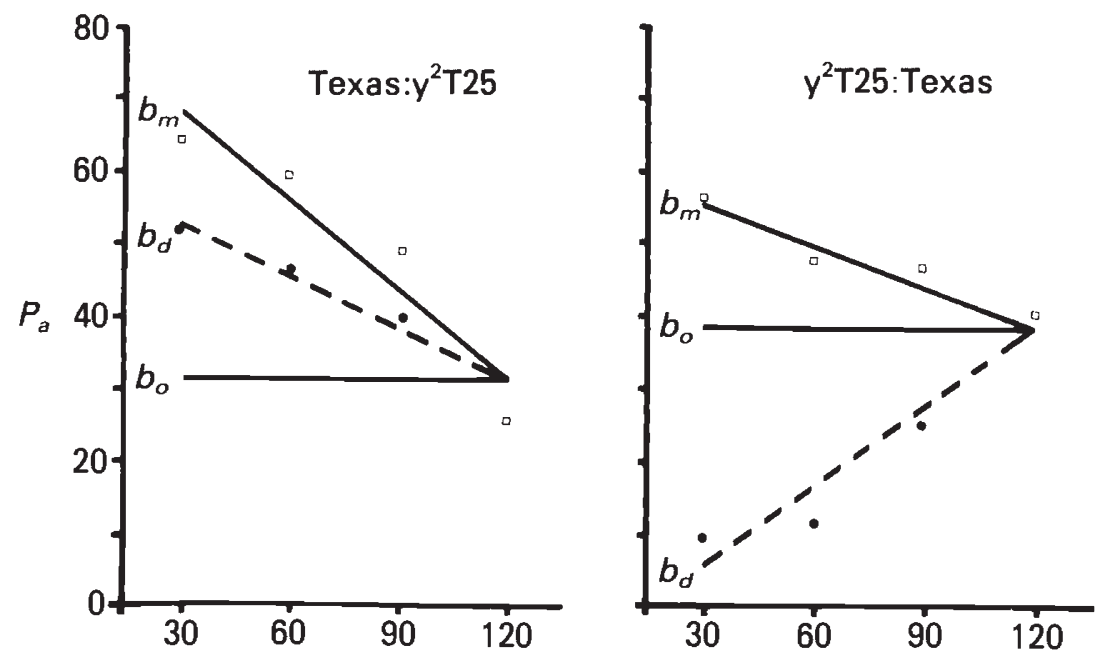

Figure 2 Estimated regression lines relating the character $\boldsymbol{P}_{a}$ to indicator genotype density for the Texas population in competition with the tester genotype $\mathrm{y}^{2} \mathrm{~T} 25$ in cycle 0 . The monoculture regressions $\left(b_{m}\right)$ are represented as solid lines and duoculture regressions $\left(b_{d}\right)$ as broken lines. The frames of reference are completed by the horizontal lines of zero relationship $\left(b_{0}\right)$. In each case the competing genotypes are represented as indicator:associate.

difference between the two slopes $\left(b_{d}-b_{m}\right)$. These two estimates are referred to as the competitive or $C$ values.

The approach of Mather and Caligari (1983), as described by Hill et al., (1986) was used to estimate the aggression and response components. Using this design, $C_{p p}=\left(-b_{m}\right)$ measures the intragenotypic effect and $C_{p y}=\left(b_{d}\right)$, measures the combined intra- and inter-genotypic effects when the indicator is $p$ (Texas population) and the associate is $y\left(\mathrm{y}^{2} \mathrm{~T} 25\right)$. Similarly, when genotype $\left(\mathrm{y}^{2} \mathrm{~T} 25\right)$ is taken to be the indicator we obtain the corresponding values $C_{y y}$ and $C_{y p}$. These four competition values and the derivations of the orthogonal parameters $a, r$ and $i$ are shown in table 1 .

The error variance against which $a, r$ and $i$ are tested was derived from $\frac{1}{4}$ of the pooled $C$-value error variance. The appropriate error variance for the $C$ values was derived from the duplicate error variance as described by Mather and Caligari (1983). The duplicate error variances throughout the experiment were based on $14 \mathrm{df}$, except in cycle 0 where 4 replicates were raised, resulting in $42 \mathrm{df}$.

In this case, and in all other analyses throughout the experiment, the fit of the linear regression model to the observed data was found to be adequate by comparing the residual variation around the regression lines with the replicate error variance.

The selection of appropriate Texas lines at each cycle of the (HR) and (LA) selection programmes was based solely on the estimates of aggression $(a)$ and response $(r)$ obtained in competition with the tester $\mathrm{y}^{2} \mathrm{~T} 25$.

Table 1 Deviations from the mean competitive value $\bar{C}$ ascribable to aggression $(a)$, response $(r)$ and their interaction $(i)$, for the genotypes $p$ (Texas population) and $y$ ( $\mathrm{y}^{2} \mathrm{~T} 25$ ) when raised in both monoculture and duoculture. The parameter estimates are given beneath the table

\begin{tabular}{|c|c|c|c|c|}
\hline & & \multicolumn{2}{|l|}{ Indicator } & \multirow[b]{2}{*}{ Mean } \\
\hline & & $p$ & $y$ & \\
\hline \multirow[t]{2}{*}{ Associate } & $\begin{array}{l}P \\
Y\end{array}$ & $\begin{array}{l}C_{p p}=\bar{C}+a+r+i \\
C_{p y}=\bar{C}-a+r-i\end{array}$ & $\begin{array}{l}C_{y p}=\bar{C}+a-r-i \\
C_{y y}=\bar{C}-a-r+i\end{array}$ & $\begin{array}{l}\bar{C}+a \\
\bar{C}-a\end{array}$ \\
\hline & \multicolumn{3}{|c|}{$\begin{aligned} \bar{C} & =\frac{1}{4}\left(C_{p p}+C_{p y}+C_{y p}+C_{y y}\right) \\
a & =\frac{1}{4}\left(C_{p p}-C_{p y}+C_{y p}-C_{y y}\right) \\
r & =\frac{1}{4}\left(C_{p p}+C_{p y}-C_{y p}-C_{y y}\right) \\
i & =\frac{1}{4}\left(C_{p p}-C_{p y}-C_{y p}+C_{y y}\right)\end{aligned}$} & $\bar{C}$ \\
\hline
\end{tabular}




\section{RESULTS}

Estimates of the regression parameters obtained throughout the experiment are shown in table $2(\mathrm{i}$ and ii) for the low aggression (LA) and high response (HR) selection programmes respectively. The values of $e$ represent the expression of the character $P_{a}$ at the reference density of 120 eggs per culture. The intra-genotypic $\left(b_{0}-b_{m}\right)$ and inter-genotypic $\left(b_{d}-b_{m}\right)$ competitive values derived from the monoculture and duoculture regression coefficients are also given.

Several important points can be made with respect to the regression parameters depicted in table 2. Although, as expected, there is considerable fluctuation in these parameters throughout the selection programme, some general trends may be identified. Where the Texas population is being selected for low aggression (table 2(i)) we might expect Texas individuals to fare progressively less well in mixtures as the selection proceeds. The estimates of $\left(b_{d}-b_{m}\right)$ show that the inter-genotypic competition exerted by Texas individuals (as associate) on the tester $\mathrm{y}^{2} \mathrm{~T} 25$ (as indicator) falls dramatically. What this means in practice is that fewer wild type individuals emerge from the duocultures. The same phenomenon is reflected in the observed increase in the inter-genotypic competition exerted by $\mathrm{y}^{2} \mathrm{~T} 25$ individuals (as associate) on Texas individuals (as indicator) even though there is a sharp decrease as the selection lines become established. Although the basic competi- tive characteristics of the tester genotype cannot have changed, it fares better in duoculture with the progressively less aggressive Texas individuals. In monocultures, however, selection of Texas individuals for lower aggression might be expected to reduce intragenotypic competition. This is seen clearly in table 2(i) where the estimates of $\left(b_{0}-b_{m}\right)$ fall from 0.41 to $0 \cdot 05$. Intra-genotypic competition among the unselected tester genotype individuals $\left(\mathrm{y}^{2} \mathrm{~T} 25\right)$ remains much more constant.

The behaviour of the competitive values in the high response (HR) selection programme (table 2(ii)) however, is more difficult to interpret. Once again we expect Texas individuals to do progressively less well in competitive situations as they become more sensitive to the prevailing competition. Furthermore, we expect the basic competitive characteristics of the tester genotype to remain unchanged. The observed values of inter-genotypic competition $\left(b_{d}-b_{m}\right)$ reveal that the competitive effect of $y^{2}$ T25 (as associate) on Texas (as indicator) is, indeed, relatively consistent throughout the selection programme. However, the ability of Texas individuals (as associate) to exert inter-genotypic competition on the tester $\mathrm{y}^{2} \mathrm{~T} 25$ (as indicator) is seen to decrease. Although the basic characteristics of the tester have not changed, the Texas individuals are progressively more sensitive to the presence of $\mathrm{y}^{2} \mathrm{~T} 25$ flies in duoculture and as a result, fewer wild type adults emerge. The situation in monoculture is less tangible. Table 2(ii) shows a substantial decrease in the levels of intra-

Table 2 Estimates of the regression parameters and competitive values obtained for the selected line at each cycle of selection (TEX S1 to TEX S6) for the low aggression (LA) and high response (HR) selection programmes (i and ii respectively). The expression of the character $P_{a}$ at the reference density of 120 eggs per culture is given as $e$. The intra- and inter-genotypic competitive values are given as $\left(b_{0}-b_{m}\right)$ and $\left(b_{d}-b_{m}\right)$ respectively

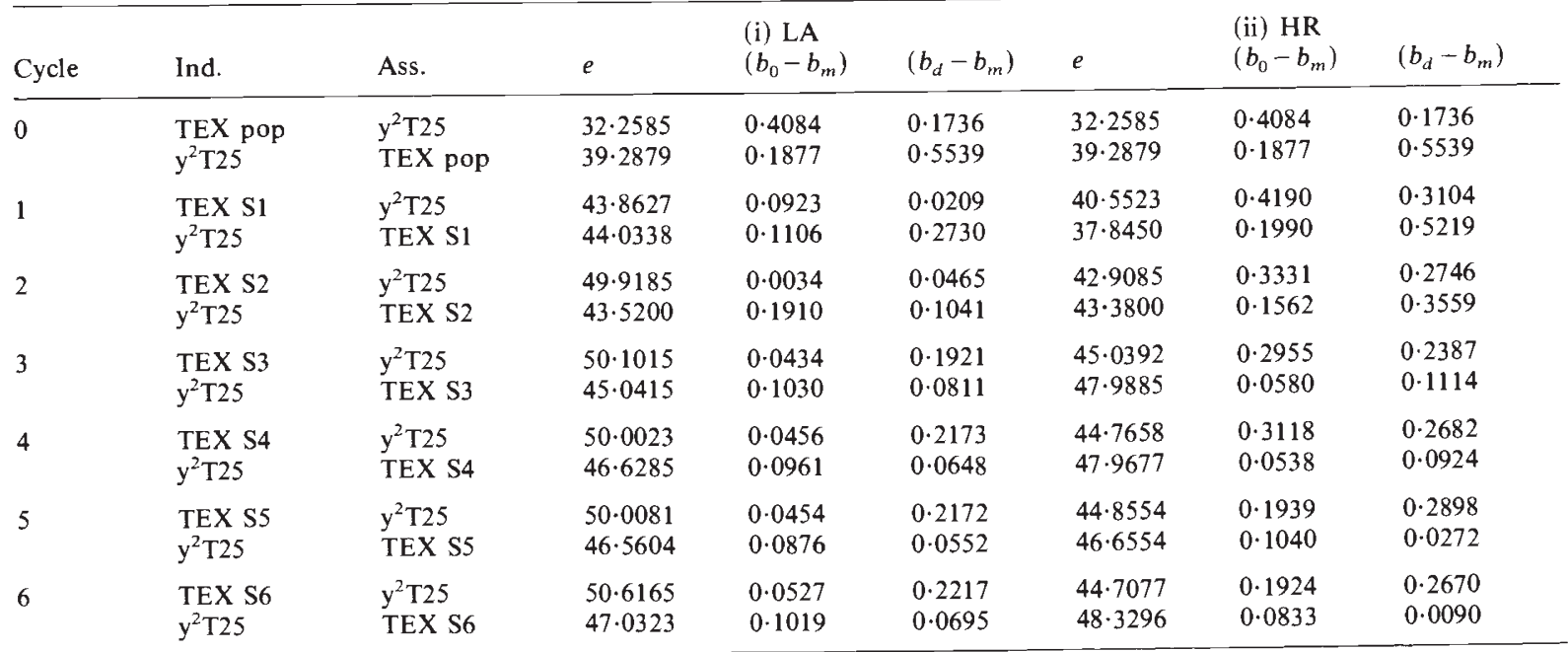


genotypic competition $\left(b_{0}-b_{m}\right)$ among Texas individuals as selection proceeds. One interpretation of this, though not entirely satisfactory, is that intra-genotypic competition decreases to compensate for increasing sensitivity to that competition. Any interpretation must, however, be made with care since the intra-genotypic competition among unselected $\mathrm{y}^{2} \mathrm{~T} 25$ individuals is also seen to decrease. If, in fact, much of the fluctuation in monoculture slopes is an experimental artefact then it serves as a very strong reminder of the importance of measuring inter-genotypic effects against the intra-genotypic yardstick.

One final point to be made with respect to table 2 concerns the $e$ values which represent the expression of the character $P_{a}$ at the reference density of 120 eggs per culture. These values are best interpreted as the basal competitive ability of the indicator genotype from which all relative intra- and inter-genotypic effects might be measured. As such, lower values of $e$ (as found for the initial segregating Texas population at cycle $0)$ reflect the intense competition which takes place. In contrast, $e$ values for inbred material are generally higher and it is clear that the selected Texas lines follow this trend. Once the selection lines become established, the $e$ values remain relatively constant with values that suggest $40-45$ per cent of the individuals competing at the reference density emerge as adults.

In order to reveal the actual progress of the selection procedures we need to consider the partition of competitive effects into aggression and response values as shown in table 3 (i and ii) for the (LA) and (HR) selection programmes respectively. These results show clearly the decrease in aggression (table 3(i)) and increase in response (table 3(ii)) of the selected Texas lines in competition with the standard tester genotype $\mathrm{y}^{2} \mathrm{~T} 25$. The results which are also depicted in fig. 3 show that aggression decreased approximately linearly for two cycles and then slowed down to the fourth cycle after which a plateau appeared to have been reached. The same figure shows that selection for high response resulted in a similar linear change during the early generations followed by a plateau from about the third or fourth generation.

As expected, the variance between the eight lines tested at each cycle reduced as selection proceeded. for both the (HR) and the (LA) programmes this reduction can be seen in the falling standard errors in table 3 and reflects the narrowing of the genetic base through inbreeding.

Several important points can be made concerning the changes in aggression and response parameters throughout the selection programme. First, changes in the selected components occur very rapidly in the early generations and this suggests that a considerable amount of additive genetic variation is involved in the control of aggression and response. This is further supported by estimates of the realised heritability taken over the whole selection programme which amount to 0.79 and 0.74 for the (LA) and (HR) selections respectively. Such rapid changes in competitive ability have been recorded previously in experiments which involve selection by, for example, Moore (1952), Pimentel et al., (1965) and Seaton and Antonovics (1967).

The general trend displayed by the LA and HR selections is for an initially rapid response during the first four cycles followed by an apparent

Table 3 Estimates of the deviations from the mean competitive value ascribable to aggression $(a)$, response ( $r)$ and their interaction ( $i$ ) for the selected line (out of eight tested) at each cycle of the (LA) and (HR) recurrent selection programmes (table $3 i$ and 3 ii respectively). The mean values of $a$ and $r$ (taken over all eight lines tested) are also given for the (LA) and (HR) programmes respectively at each cycle of selection. The standard errors appropriate to these estimates of $a, r$ and $i$ (for $14 \mathrm{df}$ ) are given and the significance of the parameters (as inferred from $t$ tests) is given as NS, $P>0.05 ;{ }^{*} 0.05>P>0.01 ; * * 0.01>P>0.001$; *** $P<0.001$

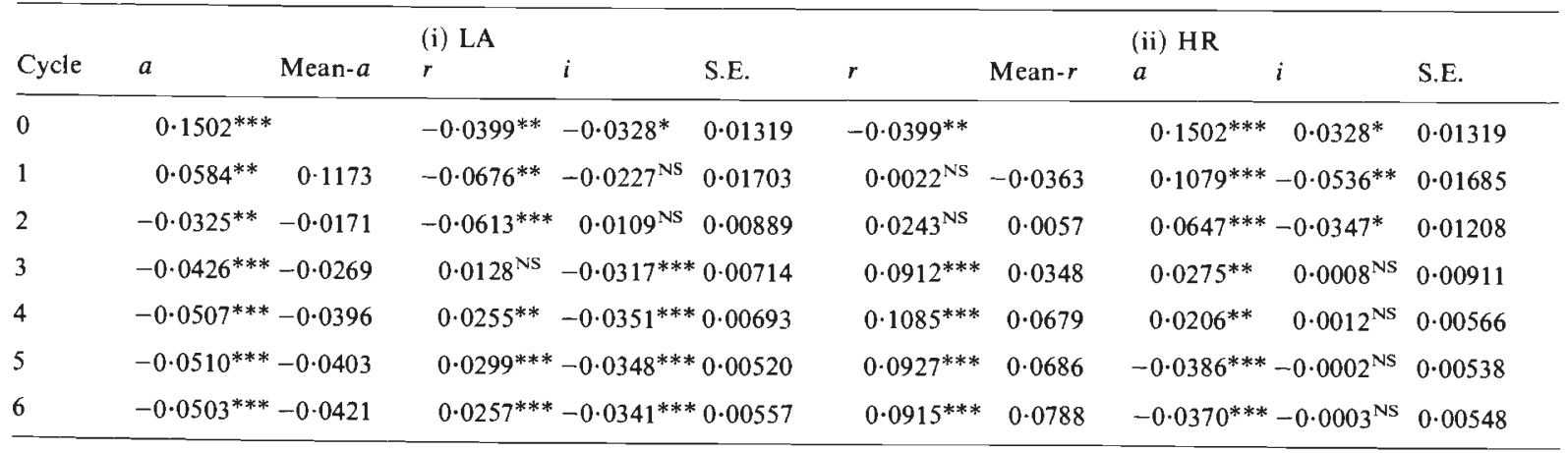




\section{Competitive Parameter}

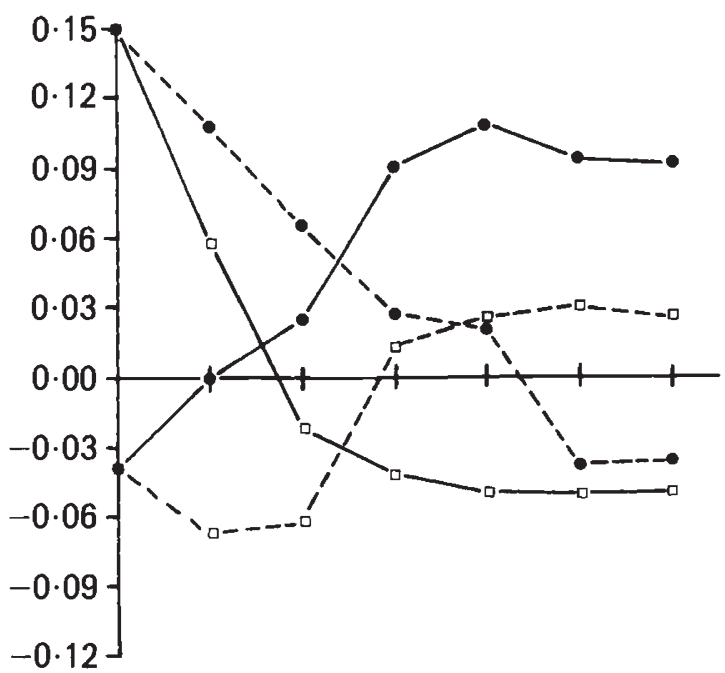

Figure 3 Progress of the HR and LA selection programmes over six cycles of selection. In the (HR) programme the response parameter $(r)$ of the selected line at each cycle is shown as a circle. The behaviour of the unselected aggression component is shown by circles linked with a broken line. In the (LA) programme the aggression parameter $(a)$ of the selected line at each cycle is shown as a square. The unselected response component being represented by squares linked by a broken line.

plateau (fig. 3). Similar trends have been observed on many occasions during selection for quantitative traits in Drosophila (Mather, 1983) and largely reflect the re-assortment of the three major chromosomes as units. Generally, after a few more generations a further response to selection is observed which is consistent with the release of genetic variability by intra-chromosome recombination. No such additional response had been obtained by the termination of these experiments at the sixth cycle of selection. This may reflect either a premature termination of the experiment or an artefact of the design. Each cycle of selection was initiated with the surviving wild type adults from the competition duocultures of the previous cycle. Thus the number of flies contributing to successive generations was necessarily limited. This level of inbreeding may have restricted the genetic variability available for selection and thereby limited the overall response. It is therefore not clear, from these experiments alone, how many loci might be involved in the determination of aggression and response.

The results in fig. 3 make it quite clear that aggression and response do not behave entirely independently. In the (HR) selection programme (table 3(ii) and fig. 3) the non-selected component (aggression) can be seen to decrease markedly and simultaneously with increasing response. It has been stated previously that variation in $r$ tends to be associated with variation in $a$, (Mather and Caligari, 1983) and in this case the two components appear to be inversely related such that selecting genes which increase response tends to bring about a decrease in aggression. In the case of the (LA) selection programme however, the changes in the unselected component $(r)$ are much less pronounced. In the first two generations particularly, there is a major decrease in aggression with no significant change in the non-selected response component. As the selected aggression component begins to plateau there is an increase in response but this is not pronounced and rapidly achieves a stable value. Any attempt to interpret these changes during artificial selection must, however, include an appreciation of the role of natural selection within this experimental design. The parents of successive generations are those which have survived competition in mixture in the previous generation. Thus, natural selection will have exerted a strong effect before the artificial selections are made and the overall changes must reflect this balance. Natural selection within the cage population of Texas has resulted in very high levels of aggression and very low levels of response (Eggleston, 1985). We might therefore expect natural selection to oppose artificial selection for low aggression and high response as practised in these experiments. The results do not provide any clear evidence for this since the selected components show rapid changes in the expected direction. Whether the changes in the non-selected components reflect the influence of natural selection, the stabilisation of aggression and response characteristics as the lines approach fixation or merely random fluctuations is difficult to determine. What is clear is that these findings tend to support those of Mather and Caligari (1983) in that variation in response tends to be associated with variation in aggression but not necessarily vice versa. The nature of the genetical control is clearly not simple and consideration of the interaction parameters reveals the different behaviour of the (LA) and (HR) selection lines.

In the case of the (HR) programme (table 3(ii)) there is little evidence of significant interaction between $r$ and $a$ components as the selection proceeds. This is in fact, the normal situation in such Drosophila experiments where interaction is rare. In the case of the (LA) programme however the interaction component tends to become very highly significant as the selection proceeds. This presumably reflects the fact that the unselected response 
component does not follow closely the behaviour of the selected aggression component, although the full implications are still under consideration.

\section{DISCUSSION}

Selection for competitive ability in Drosophila melanogaster has been rarely studied and the few documented experiments must be viewed with caution because of the lack of adequate control in the competitive environment, namely the supply of food and larval density.

The results from this experiment reveal that the Texas population of $D$. melanogaster contains extensive additive variation for two components of the competitive interaction, namely, aggression and response and that these characters may be adjusted by the application of recurrent selection against a standard tester genotype.

The speed with which the changes in response and aggression occurred in both the (LA) and (HR) selection programmes was surprising, but reflects both the high levels of additive genetic variation for these characters and the fact that the selection intensity was quite high. The selection of just one out of eight lines tested at each cycle of selection constitutes, of course, a truncation selection scheme involving the top 12 per cent in each case.

It is obvious from the results of the (HR) selection programme (table 3(ii)) that the increase in response throughout the selection programme is associated with a decrease in aggression. In contrast, the selection programme for low aggression (table 3(i)) reveals that the unselected component (response) does not show any significant change in the first two cycles, despite the dramatic reduction in the aggression component. Although the unselected response component does increase in cycle 3 , it is clear that the variation in $r$ does not closely follow that in the selected component $a$ either directly or in an inverse relationship as found for the (HR) programme.

Two interpretations of the genetical control of aggression and response have been advanced previously (Eggleston, 1985). These characters are either under independent genetical control or else are determined by a single array of genes with pleiotropic effects such that all possible combinations of aggression and response could be determined by selecting the appropriate genes. The almost symmetrical inverse relationship between the selected component $(r)$ and the unselected component $(a)$ in the (HR) programme clearly does not favour the interpretation of independent genetical control. It would appear, therefore, that these results support the hypothesis of a single array of genes responsible for the determination of aggression and response in Drosophila. The changes observed in the selected and unselected components might then be explained by postulating that some of these genes have a much larger effect on one character (aggression) than they do on the other (response). This interpretation is consistent with the variety of relationships between $a$ and $r$ described by Eggleston (1985) and Mather and Caligari (1983). Clearly, these relationships reflect the differing gene arrays fixed in each of the highly inbred lines involved in those studies.

It is not possible, however, to make any clear inference about the relative roles of natural and artificial selection in the experiments reported here. A modified experimental design may allow the two roles to be separated but would necessarily involve rounds of random mating in between cycles of selection. This may improve the long term response by resolving linkage arrangements through recombination and thereby releasing variability. The disadvantage would be that the experiments would take much longer to complete. Further clarification of the nature of the genetical control of aggression and response must await the results of experiments now in progress.

Acknowledgements We are grateful to Joachim Rodrigues de Miranda for helpful discussion and to Lynda Mitchell for technical assistance.

\section{REFERENCES}

BARNES, B. W. 1968. Stabilizing selection in Drosophila melanogaster. Heredity, 23, 433-442.

BREESE, E. L. AND HILL, J. 1973. Regression analysis of interactions between competing species. Heredity, 31, 181-200.

CALIGARI, P. D. S. 1980. Competitive interactions in Drosophila melanogaster. I. Monocultures. Heredity, 45, 219-231.

DE MIRANDA, J. R. AND EGGLESTON, P. 1987. A comparison of substitution and addition designs for the analysis of competitive interactions in Drosophila melanogaster. Heredity, 58, 279-288.

DE WIT, C. T. 1960. On competition. Versl. Landbouwk. Onderz. Ned., 66, 1-82.

EGGLESTON, P. 1985. Variation for aggression and response in the competitive interactions of Drosophila melanogaster. Heredity, 54, 43-51.

EGGLESTON, P. 1987. The use of genetic markers in the analysis of competitive interactions in Drosophila melanogaster. Genetica (in press).

FISHER, R. A. AND YATES, F. 1963. Statistical Tables for Biological, Agricultural and Medical Research, 6th edition. Oliver and Boyd, Edinburgh.

GALE, J. S. 1964. Competition between three lines of Drosophila melanogaster. Heredity, 19, 681-699. 
HILL, J., MATHER, K., CHIRWA, R. M. AND CALIGARI, P. D. S. 1986. The analysis of competitive ability in forage crops. Proceedings of the Sixth Meeting of the Eucorpia Section; Biometrics in Plant Breeding, pp. 193-204.

LERNER, I. M. AND HO, F. K. 1961. Genotype and competitive ability in Tribolium species. Am. Nat., 95, 329-343.

MATHER, K. 1983. Response to selection. In Ashburner, M., Carson, H. L. and Thompson, J. N. Jnr. (eds.) The genetics and biology of Drosophila, Vol. 3c. Academic Press, pp. 152-215.

MATHER, K. AND COOKE, P. 1962. Differences in competitive ability between genotypes of Drosophila. Heredity, 17,381407.

MATHER, K. AND CALIGARI, P. D. S. 1981. Competitive interactions in Drosophila melanogaster. II. Measurement of competition. Heredity, 46, 239-254.

MATHER, K., Hill, J. AND CAligari, P. D. S. 1982. Analysis of competitive ability among genotypes of perennial ryegrass. Heredity, 48, 421-434.
MATHER, K. AND CAligari, P. D. S. 1983. Pressure and response in competitive interactions. Heredity, 51, 435-454.

MOORE, J. A. 1952. Competition between Drosophila melanogaster and Drosophila simulans. II. The improvement of competitive ability through selection. P.N.A.S., 38 , 813-817.

PIMENTEL, D., FEINBURG, E. H., WOOD, P. W. AND HAYES, J T. 1965. Selection, spatial distribution and the coexistence of competing fly species. Am. Nat., 99, 97-109.

POWELL, W., CALIGARI, P. D. S. AND THOMAS, W. T. B. 1985. The effects of competitive interactions on breeding strategies in spring barley. Proceedings of the Sixth Meeting of the Eucorpia Section. Biometrics in Plant Breeding, pp. 243-257.

SAKAI, K. AND GOTOH, K. 1955. Studies on competition in plants. IV. Competitive ability of $F_{1}$ hybrids in barley. Heredity, 22, 19-33.

SEATON, A. P.C. AND ANTONOVICS, J. 1967. Population interrelationships. I. Evolution in mixtures of Drosophila mutants Heredity, 22, 19-33. 